\title{
Weaning from ventricular assist device support after recovery from left ventricular failure with or without secondary right ventricular failure
}

\author{
Michael Dandel ${ }^{1}$, Mariano Francisco del Maria Javier ${ }^{2}$, Eva Maria Javier Delmo ${ }^{3}$, Matthias Loebe $^{4}$, Roland Hetzer ${ }^{2}$ \\ ${ }^{1}$ Department of Cardiology, Cardio Centrum Berlin, Berlin, Germany; ${ }^{2}$ Department of Cardiothoracic and Vascular Surgery, Cardio Centrum Berlin, \\ Berlin, Germany; ${ }^{3}$ Charité-Universitätsmedizin Berlin, Charité Research Organization, Berlin, Germany; ${ }^{4}$ Thoracic Transplant and Mechanical \\ Support, Miami Transplant Institute, Memorial Jackson Health System, University of Miami, Miami, Florida, USA \\ Contributions: (I) Conception and design: All authors; (II) Administrative support: None; (III) Provision of study materials or patients: All authors; (IV) \\ Collection and assembly of data: All authors; (V) Data analysis and interpretation: All authors; (VI) Manuscript writing: All authors; (VII) Final approval \\ of manuscript: All authors. \\ Correspondence to: Michael Dandel, MD, PhD. Department of Cardiology, Cardio Centrum Berlin, Berlin, Germany. Email: mdandel@aol.com.
}

\begin{abstract}
Although complete myocardial recovery after ventricular assist device (VAD) implantation is rather seldom, systematic search for recovery is worthwhile because for recovered patients weaning from VADs is feasible and can provide survival benefits with long-term freedom from heart failure (HF) recurrence, even if a chronic cardiomyopathy was the primary cause for the drug-refractory HF necessitating left ventricular (LVAD) or biventricular support (as bridge-to-transplantation or definitive therapy) and even if recovery remains incomplete. LVAD patients explanted for myoacardial recovery compared to those transplanted from LVAD support showed similar survival rates and a significant proportion of explanted patients can achieve cardiac and physical functional capacities that are within the normal range of healthy controls. In apparently sufficiently recovered patients, a major challenge remains still the pre-explant prediction of the weaning success which is meanwhile reliably possible for experienced clinicians. In weaning candidates, the combined use of certain echocardiography and right heart catheterization parameters recorded before VAD explantation can predict post-weaning cardiac stability with good accuracy. However, in the absence of standardization or binding recommendations, the protocols for assessment of native cardiac improvement and also the weaning criteria differ widely among centers. Currently there are still only few larger studies on myocardial recovery assessment after VAD implantation. Therefore, the weaning practice relies mostly on small case series, local practice patterns, and case reports, and the existing knowledge, as well as the partially differing recommendations which are based mainly on expert opinions, need to be periodically systematised. Addressing these shortcomings, our review aims to summarize the evidence and expert opinion on the evaluation of cardiac recovery during mechanical ventricular support by paying special attention to the reliability of the methods and parameters used for assessment of myocardial recovery and the challenges met in both evaluation of recovery and weaning decision making.
\end{abstract}

Keywords: Dilated cardiomyopathy; heart failure; myocardial recovery; echocardiography; ventricular function; ventricular assist devices (VADs)

Submitted Feb 25, 2020. Accepted for publication Aug 20, 2020.

doi: $10.21037 / \mathrm{cdt}-20-288$

View this article at: http://dx.doi.org/10.21037/cdt-20-288

\section{Introduction}

The use of ventricular assist devices (VADs) is a life-saving procedure initially designed as a bridge-to-transplantation (BTT) in case of drug-refractory end-stage heart failure (HF). However, already 25 years ago it was observed that, in some 
patients, a BTT can turn into a bridge-to-recovery (BTR) allowing device explantation. Later, with the increasing use of VADs as definitive therapy it was found that also in some of those patients VADs can become a BTR. In apparently sufficiently recovered patients, a major challenge was the pre-explant prediction of the weaning success. Meanwhile this is reliably possible $(1,2)$. An open question remained the potential feasibility of VAD implantation primarily designed as a treatment strategy for myocardial recovery. Such, possibly future indication for VAD therapy, however will require a reliable pre-implantation prediction of postimplantation myocardial recovery.

The goal of this review is to offer an updated overview on the current state of knowledge on the relevance of VADpromoted cardiac improvement in order to answer the question as to whether myocardial recovery can be indeed a realistic therapeutic goal.

\section{Myocardial reverse remodeling and functional improvement in VAD recipients}

\section{Pathophysiological relationships between VAD support and myocardial recovery}

Ventricular unloading-induced lowering of myocardial wall stress and improvement of blood flow to vital organs during VAD support can abolish most of the pathophysiological triggers for myocardial remodeling and interrupt the vicious circle of ventricular dilation and reduction of the myocardial contraction efficiency (3). These beneficial effects facilitate reverse remodeling, which occasionally provide support for relevant reversal of ventricular structural and functional alterations permitting the removal of the VAD even in patients with long-lasting ventricular dilation and myocardial dysfunction before VAD implantation.

Although significant reverse remodeling with recovery of major alterations in the HF phenotype was often observed after VAD support, in most instances reversal of the HF phenotype was incomplete and this explains why sufficient and durable cardiac recovery permitting VAD explantation is relatively rare (3-6). Although reverse remodeling of the ventricular myocardium is decisive for its functional improvement, it results only rarely in a clinically relevant remission of heart function (5). However, both the individual parts of the reverse remodeling process which are essential for myocardial recovery and the minimum amounts of reverse remodeling necessary to allow VAD explantation are still badly known.
Clinically relevant cardiac recovery seems to be linked to patient age, etiology and duration of the HF as well as the amount of myocardial fibrosis prior to VAD insertion $(1,5,7,8)$. Acute forms of HF (e.g., postcardiotomy- and viral myocarditis-related HF) are more frequently and also often completely reversible during VAD support $(5,6,9)$. However, in such cases, reversible causal factors for myocardial injury usually play a key pathogenic role and the contribution of VADs to myocardial recovery is especially indirect (i.e., vital circulatory support during the time needed for spontaneous and/or medication-facilitated cardiac recovery) (3). Conversely, for patients with acute decompensated chronic HF necessitating VAD support, long-running unloadinginduced reduction of ventricular wall-tension can have decisive myocardial recovery-promoting effects.

\section{Historical overview of the major steps toward the recognition of VADs as a potential BTR}

VAD explantations were initially performed almost exclusively in left ventricular assist device (LVAD) recipients with acute forms of HF (9-12). This is not surprising because acute forms of HF are more often and even more completely reversible during ventricular mechanical support and, until quite recently, cardiac remodeling processes in patients with chronic HF were considered to be irreversible. Until the early 1990s the general opinion was that even for acute HF, clinically relevant cardiac recovery allowing LVAD explantation is highly unlikely in patients needing more than 15 days of support. Thus, in an early study from the Osaka Cardiovascular Center including 11 weaned patients with acute HF, all 6 patients who were weaned after LVAD support of 15 to 21 days died within 2 weeks, whereas the other 5 patients who could be weaned within the fist 15 days of LVAD support survived with a well maintained LV function (10). In 1994, the same study group reported the first successful elective weaning of 2 patients with an acute form of HF who needed 26 and 89 days of LVAD support, respectively (9). At publication time of the weaning results, those patients were free from HF recurrence at 9 and 10 months, respectively (9). In 1994 a study from the Texas Heart Institute (THI) revealed that prolonged LVAD support can reverse LV dilation and improve its ejection fraction (EF) in both idiopathic and ischemic cardiomyopathy but none of those patients underwent LVAD explantation (13). In 1996 the same group published the anatomic, physiologic, hemodynamic, histologic, and biochemical data of 31 LVAD recipients 


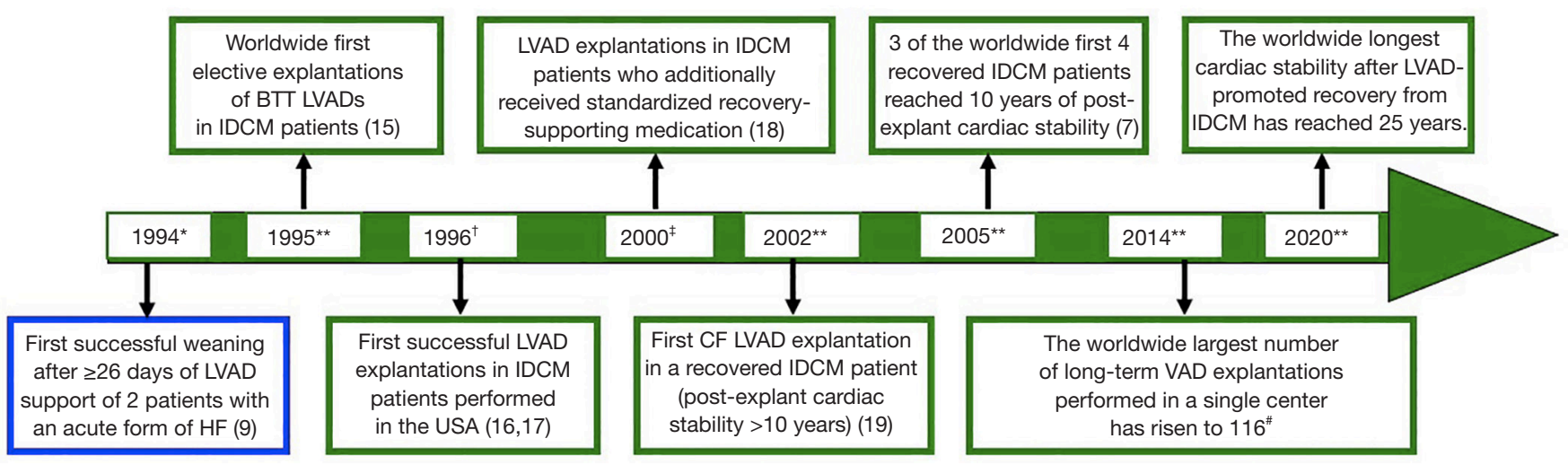

Figure 1 Timeline showing some of the major progresses and achievements in the selective use of LVADs as a BTR. *, Osaka Cardiovascular Center; ${ }^{* *}$, German Heart Center Berlin; ${ }^{\dagger}$, Texas Heart Institute and Columbia Presbyterian Medical Center; ${ }^{\ddagger}$, Royal Brompton \& Harefield NHS Foundation Trust, London; *, 102 LVADs and 14 biventricular assist devices primarily designed as BTT which were explanted in the German Heart Center Berlin since 1995 (chronic non-ischemic cardiomyopathy was the underlying cause for VAD implantation in 63 of those patients). BTT, bridge-to-transplantation; LVAD, left ventricular assist device; IDCM, idiopathic dilated cardiomyopathy; HF, heart failure; CF, continuous flow; US, United States of America; VAD, ventricular assist device. With Ref. (7,9,15-19).

(17 with idiopathic and 14 with ischemic cardiomyopathy) collected before LVAD implantation, in the course of LVAD support and at the time of heart transplantation (HTx) just before removal of the failing heart together with the LVAD (14). They concluded that some of those transplanted patients would possibly have been also weaning candidates (14).

As shown in Figure 1, the worldwide first elective LVAD explantations in patients with idiopathic dilated cardiomyopathy (IDCM) were performed in 1995 in the German Heart Center Berlin in 4 male patients (age $47 \pm 8$ years, duration of $\mathrm{HF}$ before LVAD implantation $3.5 \pm 0.9$ years, LV end-diastolic diameter $70 \pm 1 \mathrm{~mm}, \mathrm{LVEF}$ $13 \% \pm 2 \%$, LVAD support duration $7.2 \pm 2.2$ months) $(15,20,21)$. One of those patients is still asymptomatic after 25 years since LVAD removal, other 2 patients survived 16.5 and 15 years, respectively, with the native heart, and 1 patient (without post-explant recurrence of $\mathrm{HF}$ ) died from sepsis, 2.6 years after LVAD explantation. Later, in 1996, the THI group weaned 2 patients with non-ischemic chronic cardiomyopathy (NI-CCM) from LVAD and at publication time (in 1999), both patients were clinically stable and free from recurrence of HF (16). Also in 1996, other 2 patients with NI-CCM were weaned from LVAD at the Columbia Presbyterian Medical Center (17). At publication time (in 1998), both patients were alive (postexplant time 1.3 and 2 years, respectively), but one needed a second mechanical support (temporary, for 2 months) because of early recurrence of $\mathrm{HF}$ (17).
At the end of 1998 the number of patients with preimplant IDCM who were weaned in Berlin rose to 19 and reached 28 at the end of $2000(22,23)$. Several years later, after the number of patients with pre-implant IDCM who were weaned by the Berlin group increased up to 38 , the Harefield group published in 2006 similarly good weaning results in 11 patients with pre-implant IDCM confirming the initially highly disputed feasibility of weaning patients with pre-implant DCM from LVADs $(7,18)$. By the end of 2011, the total number of patients with non-ischemic DCM who were weaned by the Berlin and Harefield groups from their long-term LVAD (initially designed as a BTT) increased up to 53 and 37 , respectively $(2,24)$. The post-explant survival rates were $72.8 \%$ and $67 \%$ at 5 and 10 years, respectively, in the Berlin study, and $73.9 \%$ at 5 and 7 years in the Harefield study $(2,24)$.

Based on the convincing weaning successes obtained in patients with NI-CCM as the underlying cause for HF necessitating VAD implantation, plus the detection of LVAD-promoted reverse remodeling also on cellular, molecular and genomic level, the false previous opinion of chronic HF irreversibility was declared invalid (2-4,15,18,23-27). The growing amount of clinical and experimental research data confirming the possibility of VAD-facilitated myocardial reverse remodeling towards clinically relevant cardiac recovery has also attracted increasing interest for more focused bench-to-bedside research aiming to get the necessary insights to reach the 
goal of a potential use of VADs also as therapy-devices for reversal of chronic HF. In this respect, the National Heart, Lung, and Blood Institute convoked recently a Working Group of experts to develop recommendations for advancing the science of cardiac recovery in the setting of VADs in order to promote its implementation also as a therapeutic tool primarily designed to induce and facilitate myocardial recovery (26).

\section{Clinical relevance of LVAD-promoted reversal of myocardial remodeling and dysfunction}

\section{Incidence of cardiac improvement allowing VAD explantation}

The clinical relevance of LVAD-promoted cardiac improvement allowing device explantation is still disputed given the high differences (ranging between 2-73\%) in the reported incidence of recovery from NI-CCM $(7,18,23,28)$. These diverging data might be explainable by the different patient selection criteria for VAD implantation and explantation applied by individual centers, differences in ventricular unloading (differences in the selection of "optimal" pump speeds) and differences in pharmacological treatment during VAD support, and also by the weaning experience of clinicians (29-37). Another reason for the more often reported low incidence of relevant recovery can be the lack of testing for recovery based of the assumption that these patients cannot recover $(38,39)$. The vast majority of programs do not routinely test for recovery or try to promote it, considering the LVAD therapy as a BTT or as permanent therapy and not as a possible BTR. Thus, most do not optimize pump speed for facilitation of reverse remodeling and even stop after VAD implant the use of drugs which facilitate cardiac reverse remodeling. By contrast, some evidence suggests that a wider and more aggressive attempt to promote and specifically look for recovery in a larger population of VADs is likely to result in a higher incidence and a broader group of patients that can recover. Thus, institutions who have focused on promoting and testing for recovery and removing the pump when patients show significant improvement in cardiac function have found substantially higher rates of recovery $(7,18,23,28,40)$.

Until today, most LVAD explantations were performed after cardiac recovery from acute myocarditis and postcardiotomy HF because ventricular reverse remodeling and improvement of pump function is usually more pronounced in such cases of acute HF $(6,11,12)$. By contrast, relevant recovery from chronic $\mathrm{HF}$ is rarer and the improvement of
LV function does usually not reach a normal level, even in patients without post-weaning HF recurrence. Therefore, only few centers have carried out a considerable number of elective devices explantation in LVAD recipients with chronic $\mathrm{HF}$ after regression of $\mathrm{LV}$ size and improvement of its contractile function $(2,7,15,18,39)$. Whereas for LVAD recipients with NI-CCM clinically relevant myocardial recovery rates of $20 \%$ appeared quite possible, the recovery rates revealed by LVAD recipients with ischemic CCM rarely exceeded $5 \%(3,36,37)$.

\section{Sustainability and efficiency of cardiac improvement after VAD explantation}

After LVAD explantation, a significant proportion of patients can achieve cardiac and physical functional capacities that are comparable to that of healthy controls $(29,30)$. Thus, up to $69 \%$ of explanted LVAD patients can achieve peak $\mathrm{O}_{2}$ consumption within the ranges of healthy controls (30).

Stability of myocardial reverse remodeling and freedom from HF recurrence after weaning from VADs initially designed as BTT or permanent therapy can reach considerable levels $(2,7,25,41)$. In weaned patients with pre-implant NI-CCM, a comprehensive Berlin study yield probability of $67 \%$ and $45 \%$ for 5 and 10 years cardiac stability after LVAD removal, respectively, although most of those patients had incomplete recovery (only 9\% had an off-pump LVEF $>50 \%$ before devise explantation) (2). In another study performed by the Harefield group, the freedom from HTx or death after LVAD explantation reached $69 \%$ at both 5 and 7 years (41). In two studies using a specific protocol to promote recovery the probability of a freedom from recurrence of HF for LVAD recipients weaned from pulsatile and continuous flow ( $\mathrm{PF}$ and $\mathrm{CF}$ ) devices reached $88.9 \%$ at 4 years and $83.3 \%$ at 3 years, respectively $(18,42)$. The probability for HF recurrence during the first 12 months after LVAD removal can be less than $15 \%$, even if IDCM was the cause of the $\operatorname{HF}(6,7,12,25,31,41,43,44)$. A comparison of longterm outcomes of weaned patients to those transplanted from LVAD support has found similar survival rates (24). The rate of HTx- or VAD-free survival for the BTR and BTT groups, respectively, was $89.9 \%, 73.9 \%$, and $73.9 \%$ and $80.4 \%, 78.3 \%$, and $78.3 \%$ at 1,3 , and 7 years, respectively (24). In an early Berlin study including 36 patients with decompensated NI-CCM-related chronic HF who were weaned from their VAD (34 LVADs and 2 BiVADs), the 5-year post-weaning survival rate (including 
also the survival after HTx or $2^{\text {nd }}$ LVAD for those with HF recurrence) reached $91.7 \%$ (45). This indicates that, although VAD-promoted cardiac recovery is rather infrequently, patients who are suited be weaned from the VAD have good perspectives for durable survival without HF recurrence $(1,7,25,29,30,46-48)$.

\section{Detection of cardiac improvement in LVAD recipients and selection of weaning candidates}

Serial echocardiography (ECHO) screenings are the first-line strategy for the detection of potential weaning candidates. In hemodynamically stable patients the screenings can get started after two to four weeks of optimal and uncomplicated LV support (49). Possible weaning candidates show sinus rhythm with quite normal heart rate, normal or only slightly enlarged end-diastolic LV diameter (LVEDD), increasing LV wall motion amplitude, no or only mild mitral and/or aortic valve (AV) regurgitation, no relevant right ventricular (RV) enlargement and no or only less than moderate tricuspid regurgitation (TR) during full LVAD support $(7,41,43)$. A steady increase in both frequency and duration of $\mathrm{AV}$ openings at a constant ventricular unloading also suggests amelioration of $\mathrm{LV}$ function $(17,28,31,50,51)$. Before evaluating cardiac recovery during short-term interruptions of LVAD support in potential weaning candidates it is helpful to carry out stepwise reductions of LV unloading under TTE supervision, to test if short-term full unloading interruptions are feasible at all and also make sense $(7,41,43,52)$. If such $\mathrm{LV}$ unloading reductions already cause complains (e.g., sweating, dizziness, etc.) and/or cardiac dysrhythmias, or if the LV size increases above the normal range, and/or the right-sided heart chambers reveal anatomical and/or functional instability (RV enlargement with decrease of its ejection, increasing TR, RA volume increase), the patient is not a weaning candidate and complete interruption of LVAD support is not advisable $(2,53)$. Nevertheless, it is reasonable to continue the close monitoring of such patients and to repeat the testing few weeks later.

\section{Evaluation of cardiac improvement in weaning candidates}

The ultimate benefit of the patient from removing a VAD depends upon a safe, accurate, and reproducible evaluation of myocardial recovery where weaning candidates are evaluated during short periods of maximum possible limitation or complete interruption of LVAD support (pump rate reduction or pump stop) to test the true underlying cardiac function $(7,15,18,41)$.

\section{Particularities, challenges and tools of recovery assessment}

Recovery assessment and weaning decision-making is mostly based on ECHO and RHC data collected in the resting state during several time-limited $(\leq 15 \mathrm{~min})$ interruptions of LVAD support (off-pump or pump turn-down to zero unloading trials) under optimal anticoagulation $(1,2,7,11,41$ 43,53-55). The time restriction for the large amount of necessary measurements during the short interruption of LV unloading results in several challenges concerning data collection and their correct interpretation regarding the prediction of long-term cardiac stability after LVAD removal.

PF-VADs enable recovery assessment during a total deactivation of the pump (real off-pump trials) $(1,7,15,41,49,54)$. Interruption of unloading by CF-VADs is more challenging because their complete stop allows backflow of blood into the $\mathrm{LV}$ which increases the $\mathrm{LV}$ size (volume overload) and reduces the systemic arterial diastolic pressure $\left(\mathrm{PA}_{\mathrm{d}}\right)$ which in turn reduces the $\mathrm{LV}$ afterload. These changes in LV pre- and afterload can induce misleading interpretations of both RHC and ECHO measurements. For CF-VADs, lowering the rotor-speed (turn-down trials) to values resulting in \pm 0 pump flow in one cardiac cycle is therefore more useful than a total deactivation of the pump $(2,7,41,43,50,55,56)$. The pump speed necessary to interrupt the LVAD support without any misguiding retrograde blood flow into the $L V$ depends on the pump design of VAD [e.g., 4,000-6,000 r/min for HeartMate II, 3,000-4,300 $\mathrm{r} / \mathrm{min}$ for HeartMate 3, and 1,800-2,200 r/min for the HeartWare HVAD] $(41,52,57)$. Pulsed wave (PW) Dopplerderived calculations of forward and reverse velocity-time integral at the inflow cannula can facilitate to obtain a zeronet flow by pump speed adjustments $(36,42,52,57)$. Also RHC hemodynamic measurements can be affected by the retrograde blood flow during a complete stop of CF-VADs. This can be avoided by occlusion of the outflow cannula with an inflated balloon during the off-pump trials $(2,58,59)$.

In Berlin, all patients who were weaned from VADs underwent recovery assessment exclusively at rest in order to avoid any detrimental impact of possible hemodynamic overloading on the often not yet finished myocardial recovery processes $(2,36,43)$. Despite of this restriction that does not allow the evaluation of cardiac adaptation to 
physical activity, the outcome of those patients after VAD explantation was by no means worse than that reported by other groups who additionally used dobutamine stress ECHO (DSE) and/or exercise testing for recovery assessment (2,19,37,41,43,52,60-62). Nevertheless, exercise stress ECHO (ESE) and off-pump DSE can provide helpful details concerning myocardial recovery $(41,60,62)$. To evaluate the $\mathrm{LV}$ inotropic reserve in patients with a HM II LVAD support who, in the resting state, remained free of symptoms during a $15 \mathrm{~min}$ pump reduction to $6,000 \mathrm{r} / \mathrm{min}$, the Harefield Hospital used additionally also a 6-min walk test with and without pump flow reduction, followed evaluation of the cardiac response by TTE (42). It is however important to obviate any myocardial overstraining which could impair the possibly still ongoing myocardial recovery processes, not only before but also early after removal of the mechanical support. This recommendation is also supported by experimental studies which revealed that after LVAD-promoted restoration of LV structure and function, recovered hearts are initially prone to hemodynamic stress, and that this vulnerability diminishes only over time (63).

Cardiopulmonary exercise testing (CPET) during reduction or interruption of LVAD support has also been used for assessment of recovery $(18,33,41,64,65)$. Given that the exercise capacity is related also to extra-cardiac factors it is not surprising that low peak oxygen consumption $\left(\mathrm{pVO}_{2}\right)$ alone cannot reliably identify persons with serious hemodynamic disturbances during exercise $(30,65)$. One study showed that CPET has only restricted ability to reflect native cardiac function in LVAD recipients (64). Nevertheless, a $\mathrm{pVO}_{2}>16 \mathrm{~mL} / \mathrm{kg} / \mathrm{min}$ might be useful as a complementary LVAD explantation criterion $(18,30)$. However, when taking into account that recovered hearts are initially prone to hemodynamic overstressing, CPET should be conducted with considerable caution and under close monitoring in LVAD recipients with incomplete recovery (63).

\section{Strategies for assessment of recovery in LVAD recipients}

ECHO (usually TTE) is the main imaging tool for assessment of recovery in LVAD recipients (Figure 2). During off-pump or pump turn-down trials, the ECHO is usually performed step-by-step in the course of several interruptions of the LVAD support for about 5-15 $\mathrm{min}$ $(2,7,56)$. The optimal duration of such discontinuations of $L V$ unloading necessary to evaluate of the amount and stability of cardiac recovery is not clearly established and depends also on the examiner's experience. However, several short successive unloading interruptions of $\leq 5 \mathrm{~min}$ are less risky for the patient than one or two longer (15-20 min) cessations of pump flow and LV support. Full pump-stop or pump turn-down to \pm 0 flow should be considered very carefully in patients after stroke or transient ischemic attack, in those with hemolysis or problems with anticoagulation therapy, and are not indicated in patients suspected for pump thrombosis (even in the absence of LVAD dysfunction) (56).

ECHO examination carried out at off-pump or pump turn-down trials should be as detailed as possible with inclusion of tissue-Doppler imaging and speckle-tracking ECHO (STE) measurements $(2,25,33,43,56,66,67)$. STE allows differentiation between active and passive movement of ventricular walls, quantification of components of myocardial contractile function that cannot be visually assessed (e.g., longitudinal myocardial shortening), and also the evaluation of intraventricular asynchrony and dyssynergy $(32,68)$. Not all ECHO variables which support the weaning decision-making process can be obtained in all patients (owing to inadequate image quality, which is more frequent in the presence of a VAD). This applies in particular for Doppler and STE measurements performed during rotor-speed reduction. Difficulties in the estimation of LV systolic function may occur in patients with low systemic diastolic arterial pressure $\left(\mathrm{AP}_{\mathrm{d}}\right)$, during the unloading interruption trials $(54,69)$. By reducing the $\mathrm{LV}$ afterload, low $\mathrm{AP}_{\mathrm{d}}$ can induce misleading overestimation of LV systolic function by increasing the $\operatorname{LVEF}(7,50)$. Accurate assessment of MR at low speed (no net flow) testing is also important because significant MR is not only a risk factor for post-explant deterioration of cardiac function but it also hampers the evaluation of LV systolic function (leads to a misleading increase in the LVEF by overestimation of the stroke volume) (70).

RHC is the next necessary step as soon as there is $\mathrm{ECHO}$ evidence of $\mathrm{LV}$ reverse remodeling and functional improvement $(2,30)$. RHC at rest, during repeated short (5-15 min) interruptions of LVAD support is also mandatory in weaning candidates before any preliminary decision-making. In patients with CF-LVADs, preliminary RHC measurements are more reliable if they are preceded by occlusion of the outflow cannula with an inflated balloon which enables full pump-stops without any misleading backflow of blood into the LV (2,58,59). Normal (possibly also borderline-normal) and also stable RHC-derived 


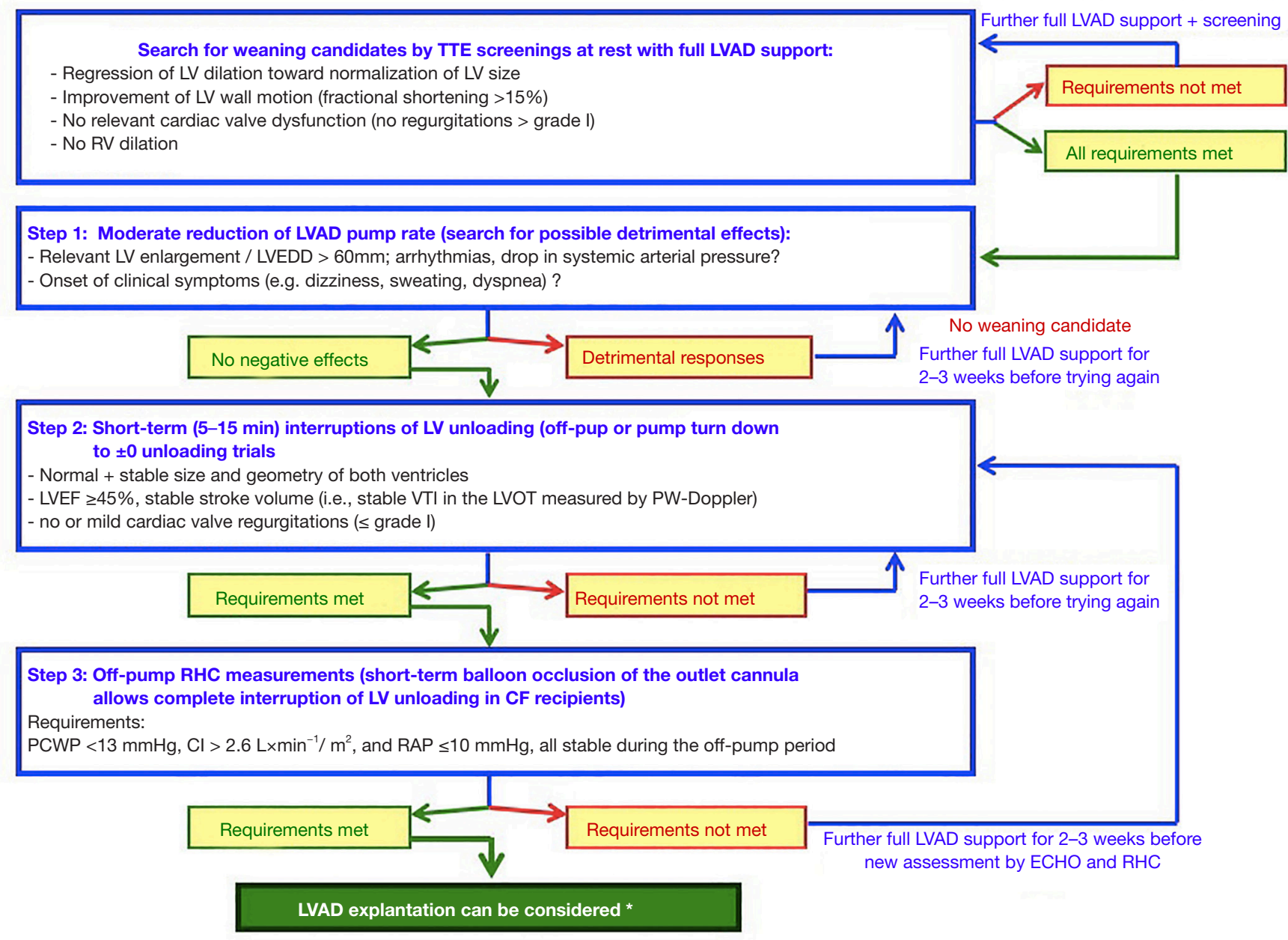

Figure 2 Evaluation of cardiac recovery in LVAD recipients based on ECHO and RHC examinations obtained in resting conditions. *, especially in patients with adequate renal, hepatic, neurologic and pulmonary function. ECHO, echocardiography; RHC, right heart catheterization; TTE, transthoracic ECHO; LVAD, left ventricular assist device; LV and RV, left and right ventricle, respectively; LVEDD, LV end-diastolic diameter; LVEF, LV ejection fraction; BSA, body surface area; TR, tricuspid regurgitation; VTI, velocity-time integral; LVOT, LV outflow tract; PW, pulsed wave; CF, continuous flow; SV, stroke volume; PCWP, pulmonary capillary wedge pressure; CI, cardiac index; RAP, right atrial pressure.

measurements obtained during off-pump trials are the basic pre-requisite for preliminary decisions in favor of a possible LVAD explantation (2). Particularly important is to make sure that the pulmonary capillary wedge pressure (PCWP) does not go up during the interruptions of LVAD support. A cardiac index $(\mathrm{CI})>2.6 \mathrm{~L} / \mathrm{min} / \mathrm{m}^{2}$ at rest, which does not drop significantly at interruption of LV support and remains stable during the $\mathrm{LV}$ unloading interruption trials, is an essential precondition for a possible weaning from the LVAD (53). In patients with borderline-normal off-pump ECHO data the CI becomes more important and CI values $\geq 2.8 \mathrm{~L} / \mathrm{min} / \mathrm{m}^{2}$ would be desirable. Other hemodynamic requirements for a possible $\mathrm{LVAD}$ explantation are offpump PCWP $<12 \mathrm{mmHg}$ and right atrial (RA) pressure <10 mmHg (2,30). RHC measurements during supine bicycle exercise testing were also performed to evaluate myocardial recovery in LVAD recipients, but their benefits for weaning decisions have not been clearly proven yet (71). In weaning candidates were LVAD explantation appears appropriate and possible, a complete interruption of LVAD support for 15-20 min in the operation room with simultaneous ECHO and hemodynamic measurements (without any use of 
inotropic agents or medication for adjustment of ventricular preload and afterload, neither before nor during the offpup trial) is mandatory for the final decision to explant the $\operatorname{LVAD}(1,2,36)$.

\section{Assessment of recovery in patients with biventricular VAD support}

\section{Recovery detection and evaluation in long-term BiVAD recipients}

Weaning from long-term biventricular assist devices (BiVADs) appeared only rarely possible, probably because of the more severe initial disease prompting the need for BiVAD support $(34,43,72)$. However, if possible, BiVAD explantation can provide outcomes quite comparable to those achieved after LVAD removal (34).

$\mathrm{ECHO}$ and RHC are also the major tools for recovery assessment after BiVAD implantation but the whole procedure is more challenging. To assess cardiac global recovery, it is necessary to interrupt the unloading of both ventricles. However, given the high susceptibility of the RV to sudden increase of its loading conditions, RV support should be interrupted about 30 seconds before the interruption of $\mathrm{LV}$ unloading $(2,34,43)$. During interruption of the BiVAD support, particular attention must be given to the RV size, geometry and loading conditions, as well as to possible changes in TR. Increase in RV end-diastolic diameter (RVEDD) and short-/long-axis ratio (L/S), as well as rise in right atrial pressure (RAP) beyond $10 \mathrm{mmHg}$ and increase in TR, indicate an inability of the RV to eject the necessary amount of blood into the pulmonary artery. The reason for that could be an inadequately recovered RV myocardial contractility or an increased resistance in the pulmonary vessels (inadequately recovered $\mathrm{LV}$ function), or also due to an inadequate myocardial recovery of both ventricles. If the PCWP is below $12 \mathrm{mmHg}$ and the PAP remains unchanged or even falls, the explanation for the right-sided heart response to the BiVAD support discontinuation is an insufficient improvement of $\mathrm{RV}$ contractility. If, by contrast, right-sided heart dilation and RV dysfunction occur simultaneously with an increase in both PCWP (up to $\geq 13 \mathrm{mmHg}$ ) and PAP, the major cause for that inadequate response to the BiVAD discontinuation is a too high RV afterload caused by a LV failure-induced increase in left-sided heart filling pressures. If in the absence of sufficient LV improvement, repeated discontinuations of only the RV support are not followed by pathologic changes neither in the right-sided heart cavities size, geometry and filling pressures, nor in tricuspid valve competence and $\mathrm{LV}$ pump-flow, a switch from BiVAD to LVAD support could be reasonable because RV improvement accompanied by reduction of $\mathrm{RV}$-supporting pump-flow raises the risk of $\mathrm{RV}$ pump thrombosis $(34,73)$.

\section{Monitoring of RV improvement in LVAD recipients with a temporary RVAD}

RV failure (RVF) is far more often reversible than LV failure and about $80 \%$ of secondary RVF cases in LVAD recipients can be reversed by a temporary RVAD (t-RVAD) $(72,74-76)$. In most cases, assessment of RV recovery can be started after 48-72 hours of t-RVAD support at optimal LV unloading by the LVAD. Clinically stable patients with sinus rhythm and quite normal heart rate, regression of RV dilation and no relevant TR, as well as improvement of RV wall motion, can be considered as candidates for possible weaning from the t-RVAD $(77,78)$. For assessment of RV recovery, the first step should be a step-by-step reduction of RVAD flow to $2 \mathrm{~L} /$ min at $0.5 \mathrm{~L} /$ day increments under ECHO control and close monitoring of hemodynamic responses (prolonged reduction of RV pump flow below $2.0 \mathrm{~L} / \mathrm{min}$ is not recommended) (77,79-81). The use of a phosphodiesterase-5 inhibitor for PVR reduction can be helpful for RV recovery assessment (79). CI decrease (or LVAD-index decrease) in response to reduction of $\mathrm{t}$-RVAD flow signalizes poor RV improvement and also the necessity to stop the recovery testing and to restore the original RVAD settings $(79,82)$. If $\mathrm{ECHO}$ and RHC parameters are not affected by moderate lowering of t-RVAD flow rate, brief interruptions (for 5-10 min) of the RVAD support or reduction to 1 litre in flow are required for the actual evaluation of RV improvement. If ECHO the parameter values continue to stay the same and RHC measurements remain within the normal range, durable RV improvement is highly probable (Figure 3). For estimation of $\mathrm{RV}$ functional reserve, it be reasonable to calculate also the $\mathrm{RV}$ load adaptation index $\left(\mathrm{LAI}_{\mathrm{RV}}\right)$ using the formula: $\mathrm{LAI}_{\mathrm{RV}}$ $=\left(V_{T I} I_{T R} \times L_{E D}\right) / A_{E D}$, where $V_{T I}$ iR is the TR velocity-time integral (corresponds to the mean pressure-gradient between the $\mathrm{RV}$ and RA during systole), $\mathrm{L}_{\mathrm{ED}}$ the end-diastolic RV long-axis length, and $\mathrm{A}_{\mathrm{ED}}$ the end-diastolic RV area (all measured in the apical 4-chamber view) $(34,83,84)$. This easily calculable index is based on the relationship between $\mathrm{RV}$ load and RV dilation (taking also the RA pressure changes into account). During discontinuation of the RVAD support, a $\mathrm{LAI}_{\mathrm{RV}}$ value $\geq 18$ indicates normal adaptability of the $\mathrm{RV}$ pump function to increased afterload and suggests that the $\mathrm{RV}$ does not require further RVAD support as long as the 


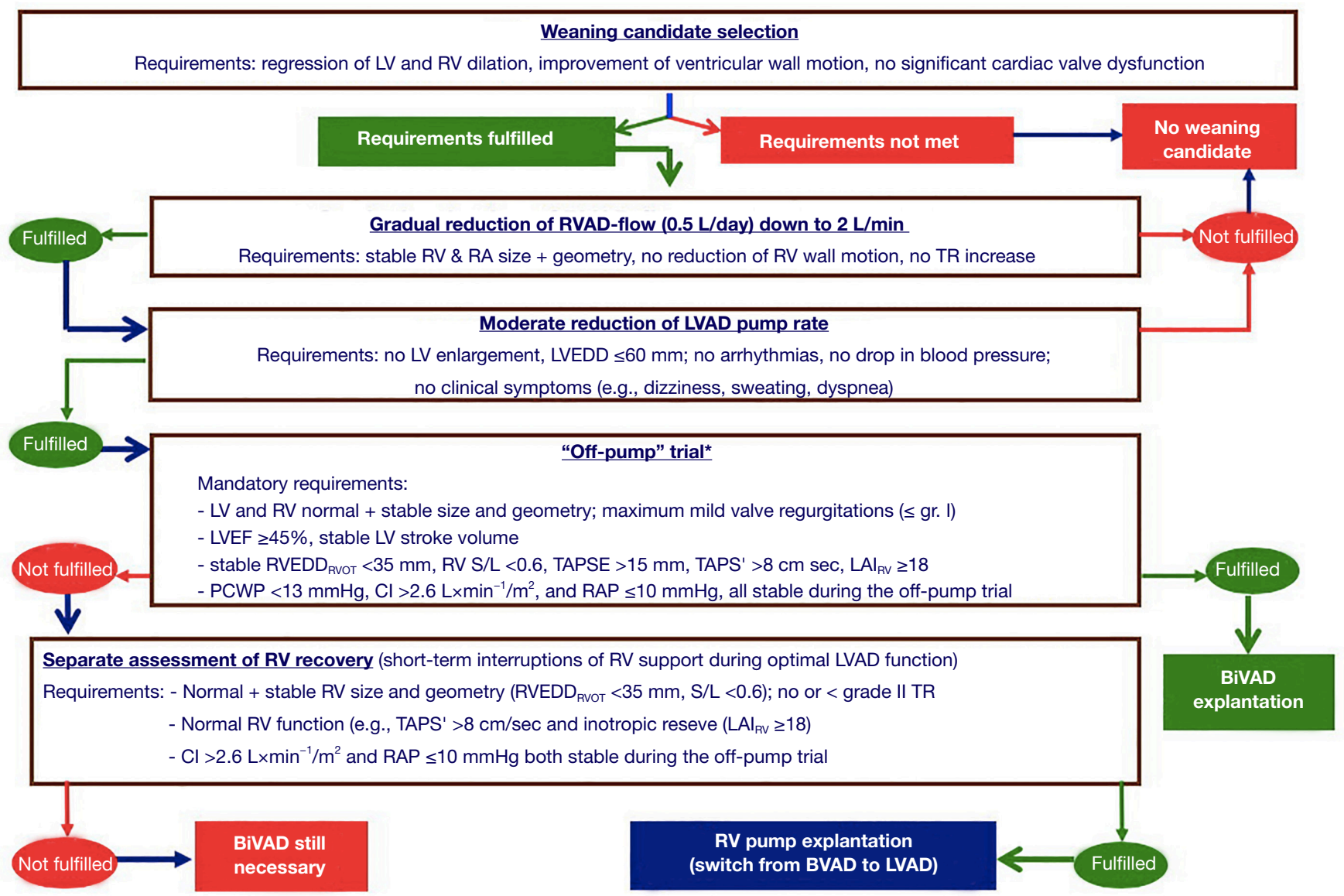

Figure 3 Evaluation of cardiac recovery in BiVAD recipients based on ECHO and RHC examinations obtained in resting conditions. *, adequate anticoagulation is mandatory; ventricular unloading interruption should be started with the RV pump ( 30 sec before interruption of the LV pump). BiVAD, biventricular assist device; ECHO, echocardiography; RHC, right heart catheterization; LV and RV, left and right

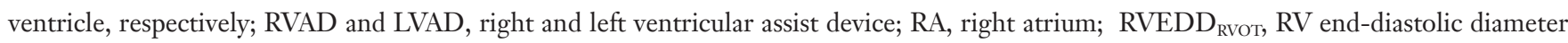
at the RV outflow tract; S/L, end-diastolic short-/long-axis ratio; PW, pulsed-wave; TAPSE and TAPS', tricuspid annulus peak systolic excursion and velocity, respectively; $\mathrm{LAI}_{\mathrm{RV}}, \mathrm{RV}$ load adaptation index; $\mathrm{VTI}_{\mathrm{LVOT}}$, velocity-time integral in the $\mathrm{LV}$ outflow tract; CVP, central venous pressure; CI, cardiac index.

resistance in the pulmonary circulation will not be excessively high $(34,83)$. However, in the absence of contraindications for a further prolongation of the RVAD support it can be helpful for weaning decision-making to prolong the RV mechanical assistance with the lowest admissible RVAD flow for up to 24 hours. If the right-sided heart size, geometry, and function continue to stay the same, RVAD removal is possible and the probability of a stable post-explant RV function can reach $90 \%$ (80). Persistently low $\mathrm{LAI}_{\mathrm{RV}}$ indicates limited ability of the RV to adapt its contractile capabilities to moderate rise of afterload, even if the PVR does not exceed the normal limits (84). Therefore, in patients with stable ECHO and RHC measurements during short-term moderate reduction of RVAD flow, but with pathological alterations during interruptions of RV unloading associated with low $\mathrm{LAI}_{\mathrm{RV}}$ values, there will be a need for further RV support because, in LVAD candidates, a pre-implant $\mathrm{LAI}_{\mathrm{RV}}$ $\leq 14$ appeared predictive for RVF during LVAD support even in patients with subsequent normalization of RV afterload (83). Therefore, even in LVAD recipients whose RV size, geometry and wall motion remain normal and stable during the short interruptions of the additional RVAD support, as long as the $\mathrm{LAI}_{\mathbf{R V}}$ does not exceed the threshold of 14 , the risk for RVF recurrence after RVAD removal 
remains relatively high. Restoration of the original RVAD settings and further continuation of full RV unloading for additional days will be thus necessary before the next assessment of RV recovery. After an unsuccessful weaning attempt, intensification of pulmonary vasodilator therapy aiming to enhance the LVAD-induced reduction of the PVR can increase the chances for a better RV function at the next evaluation of RV recovery. The use of inotropic medication before and during the assessment of RV improvement can be misleading and should therefore be avoided.

\section{Weaning decision-making in VAD recipients}

\section{Pre-explant predictors for post-explant stability of cardiac improvement}

ECHO parameters along with RHC data obtained during the short-term reductions or interruptions of VAD support enable identifying patients with good chances to remain free from recurrence of HF over many years after VAD explantation, even if IDCM was the primary cause for the HF requiring a life-sustaining VAD support (7). This is especially true if the stability of ECHO parameters during and between the VAD discontinuation trials performed after the maximum LV improvement has been reached, is also taken into consideration $(1,2,58)$. Because the most optimal duration of VAD support for the achievement of the maximum possible improvement varies widely from one patient to the other, the VAD should be removed in recovered patients only after the follow-up off-pump tests showed no further cardiac improvement (43).

Prediction of post-explant freedom from HF recurrence is challenging (Figure 4) due to the complexity of the factors involved. Both ECHO and invasive hemodynamic measurements are alone not sufficiently predictive for post-weaning long-term cardiac stability (2). Only the interconnected utilisation of ECHO- and RHC-derived parameters allows such a prediction with appropriate reliability. Thus, under resting conditions, pre-explant offpump LVEF $\geq 45 \%$ in weaning candidates with normal off-pump hemodynamic confirmed by RHC, revealed only a moderate predictive value of $74 \%$ for $\geq 5$-year postweaning freedom from HF recurrence $(1,2)$. Nevertheless, combined with either HF duration of $\leq 5$ years prior to LVAD implantation or normal off-pump LV end-diastolic size and/or geometry before LVAD explantation, or preexplant LV systolic wall motion peak velocity $\geq 8 \mathrm{~cm} / \mathrm{s}$, the predictive value of $\mathrm{LVEF} \geq 45 \%$ can exceed $85 \%(1,2)$.
Taking into calculation also the pre-explant stability of $\mathrm{LV}$ size, geometry and EF after the LV recovery has reached its maximum level, as well as their stability during the final offpump trial before LVAD explantation, the predictive value of ECHO-derived parameters for $\geq 5$ years post-weaning freedom from $\mathrm{HF}$ recurrence can exceed $90 \%(2,85)$. DSE and ESE were also used in smaller studies for pre-explant prediction of cardiac stability after LVAD explantation but the benefit-risk relationship has not yet been established $(37,41,60)$. A LVEF $\geq 53 \%$ after the $6 \mathrm{MW}$ was also indentified as a predictor of recovery with sensitivity and specificity of $93 \%$ and $80 \%$, respectively $(54,57)$. However, a $6 \mathrm{MW}$ with interruption of ventricular unloading is risky.

\section{Main risk factors for reappearance of heart failure after weaning from VAD}

In clinically stable VAD recipients with relevant ventricular reverse remodeling and functional improvement several risk factors for HF reappearance after VAD removal, which can be detected by ECHO and RHC examinations, must be taken into consideration before taking the final decision to explant the VAD (Figure 4). A pre-explant LVEF $<45 \%$, as well as an unstable LVEF (i.e., LVEF reduction after the maximum recovery has been achieved and/or LVEF decrease during the final pre-explant off-pump trial), even if it finally remains above $45 \%$, revealed high predictive values (up to $90 \%$ ) for HF reappearance during the first 3 years after weaning $(1,2)$. In patients with pre-explant offpump LVEF $\geq 45 \%$, but without normalization of $L V$ size and geometry (indicating incomplete reverse remodeling), or unstable LV geometry in the course of the last preexplant off-pump trial, the probability of HF reappearance during the first 3 years after LVAD explantation can attain values of up to $89 \%(1,2,69) . \mathrm{A} \mathrm{AP}_{\mathrm{d}} \leq 50 \mathrm{mmHg}$ during the off-pump or pump turn-down trials is also a risk factor for post-explant HF recurrence even in patients who had a pre-explant LVEF $\geq 45 \%(7,50,69)$. An increase in resting heart rate by $>25 \%$ and/or new onset or increase in the number of extrasystoles during the mechanical unloading interruptions are also risk factors for $\mathrm{HF}$ recurrence after LVAD removal $(2,36,43)$. PCWP increase above 13 $\mathrm{mmHg}$, reduction of cardiac output by $>15 \%$, as well as a mean RA pressure $>10 \mathrm{mmHg}$ or its increase of more than $50 \%$ during off-pump RHC measurements performed under resting conditions, are major risk factors for postweaning HF recurrence $(2,41,64)$. Long-lasting ( $>5$ years) pre-implant $\mathrm{HF}$ is another risk factor for HF recurrence 


\begin{tabular}{|c|c|c|c|}
\hline $\begin{array}{l}\mathrm{ECHO} \text { and } \mathrm{RHC} \text { data obtained at } \\
\text { rest during pre-explant off-pump } \\
\text { trials* }^{*}\end{array}$ & $\begin{array}{l}\text { PV for } \geq 5 \text { years cardiac } \\
\text { stability }\end{array}$ & Risk factors for post-explant HF recurrence & $\begin{array}{l}\text { PV for HF recurrence } \\
\text { after } \leq 3 \text { years }\end{array}$ \\
\hline $\begin{array}{l}\text { LVEF } \geq 45 \% \text { and normal LVEDD at } \\
\text { the last off-pump }{ }^{\dagger} \text { trial }\end{array}$ & $86 \%$ ** & $\begin{array}{l}\text { LVEF } 35-45 \% \text { at the last pre-explant off- } \\
\text { pump }{ }^{\dagger} \text { trial in patients with pre-implant HF } \\
\text { duration of }>5 \text { years }\end{array}$ & Up to $100 \%$ \\
\hline LVEF $\geq 45 \%$ and $\mathrm{RWT}_{\mathrm{LV}} \geq 0.38$ & $87 \%$ ** & $\begin{array}{l}\text { Unstable LVEF } \geq 45 \% \text { (pre-explant alteration of } \\
>10 \% \text { of best) }\end{array}$ & $90 \%$ \\
\hline Stable $^{\dagger \dagger}$ LVEF $\geq 45 \%$ & $86 \%$ ** & $\begin{array}{l}\text { No LVEDD normalization plus persistence of } \\
\text { LV geometry alterations }\left(\mathrm{RWT}_{\mathrm{LV}}<0.38\right) \text { despite } \\
\text { of optimal } E F(L V E F \geq 45 \%)\end{array}$ & $\begin{array}{l}89 \% \text { and } 82 \%, \\
\text { respectively }\end{array}$ \\
\hline $\begin{array}{l}\text { Stable }^{\dagger \dagger} \text { LVEF } \geq 45 \% \text { plus normal } \\
\text { and stable LVEDD }\end{array}$ & $94 \%$ ** & $\begin{array}{l}\text { LVEF } \geq 45 \% \text { with reduced or unstable wall } \\
\text { motion velocity }(\mathrm{Sm}<8 \mathrm{~cm} / \mathrm{s} \text { or } \mathrm{Sm} \text { alteration } \\
\text { of }>10 \% \text { during the last off-pump }{ }^{\dagger} \text { trial) }\end{array}$ & $\begin{array}{l}83 \% \text { and } 90 \%, \\
\text { respectively }\end{array}$ \\
\hline $\begin{array}{l}\text { Stable }^{\dagger \dagger} \text { LV SV (i.e., stable PW } \\
\text { Doppler-derived VTI in the LVOT) } \\
\text { Absence or } \leq \text { grade } 1 \text { AR and/or } \\
\text { MR }\end{array}$ & \multirow{2}{*}{$\begin{array}{l}\text { All are required pre- } \\
\text { conditions for successful } \\
\text { weaning } \\
\text { Alone not predictive for } \\
\text { long-term freedom from } \\
\text { HF recurrence after LVAD } \\
\text { explantation }\end{array}$} & $\begin{array}{l}\text { SV reduction (i.e., VTI reduction in the LVOT) } \\
\text { during the last off-pump }{ }^{\dagger} \text { trial. } \\
\text { Relevant LV diastolic stiffness despite optimal } \\
\text { LVEF ( } \geq 45 \%)\end{array}$ & \multirow{3}{*}{$\begin{array}{l}\text { All are validated } \\
\text { risk factors for early } \\
\text { recurrence of HF after } \\
\text { LVAD removal. Currently } \\
\text { there are no accurate } \\
\text { figures available for } \\
\text { their predictive value for } \\
\text { early recurrence of HF } \\
\text { after LVAD removal }\end{array}$} \\
\hline $\begin{array}{l}\text { No RV dilation (RVOT-EDD }<35 \\
\mathrm{~mm} \text { and short/long axis-ratio }<0.6 \\
\text { Absence of or }<\text { grade } 2 \text { TR and/ } \\
\text { or PR }\end{array}$ & & $\begin{array}{l}\text { LVEF } 45-50 \% \text { with concurrent MR grade I-II } \\
\text { (possible misleading overestimation of LVEF) } \\
\text { Systemic } \mathrm{AP}_{\mathrm{d}} \leq 50 \mathrm{mmHg} \text { (possible } \\
\text { overestimation of LVEF) }\end{array}$ & \\
\hline $\begin{array}{l}\text { RAPm }<10 \mathrm{mmHg} \text { during the last } \\
\text { off-pump }{ }^{\ddagger} \text { trial }\end{array}$ & $\begin{array}{l}\mathrm{RHC} \text { data alone cannot } \\
\text { predict post-weaning cardiac } \\
\text { stability, but the limit values } \\
\text { are required preconditions } \\
\text { for successful weaning }\end{array}$ & $\begin{array}{l}\text { TR (new appearance or accentuation) with or } \\
\text { without increase in TR jet velocity during the } \\
\text { last off-pump }{ }^{\dagger} \text { trial }\end{array}$ & \\
\hline
\end{tabular}

Figure 4 Pre-explant prediction of post-explant cardiac stability in LVAD recipients and major risk factors for recurrence of heart failure after LVAD explantation $(1,2,7,18,19,30,36,41,43,66,69) .{ }^{*}$, off-pump trials were conducted under resting conditions, without any inotropic myocardial support; **, the predictive value of transthoracic ECHO parameters for $\geq 5$ years freedom from post-explant HF recurrence is valid only for patients with normal off-pump hemodynamic RHC measurements before explantation; ${ }^{\dagger}$, off-pump trials for ECHO measurements implied either short-term pump stops (true off-pump trial) or short-term pump rate reductions to values resulting in \pm 0 flow in one cardiac cycle, depending on whether the LVAD is a pulsatile-flow or a continuous-flow (CF) pump, respectively; ${ }^{\dagger+}$, no alteration after maximum recovery between and during further pre-explant follow-up off-pump controls; ${ }^{\ddagger}$, in CF LVAD recipients, hemodynamic parameter measurements were obtained during repeated pump stops with simultaneous balloon occlusion of the outflow graft; *, RV load adaptation index (LAIRV) values between 14-18. LV and RV, left and right ventricle, respectively; LVAD, left ventricular assist device; ECHO, echo-cardiography; RHC, right heart catheterization; PV, predictive value; HF, heart failure; LVEF, LV ejection fraction; LVEDD, LV end-diastolic diameter; BSA, body surface area; RWTLV, LV relative wall thickness ([posterior wall thickness + ventricular septum thickness]/LVEDD); S/LED, end-diastolic short/long axis ratio measured in the apical $4 \mathrm{C}$, four chamber view; Sm, peak systolic wall motion velocity (measured at the basal posterior. 
after LVAD explantation, particularly in patients who need more than 6 months of LVAD support to achieve maximal possible cardiac improvement or in those with borderline $\mathrm{ECHO}$ and/or invasive hemodynamic measurements during the final off-pump/pump turn-down trials $(1,43,47)$.

\section{Elective VAD implantation as a bridge to recovery}

Reliable pre-implant prediction of sustained myocardial recovery during MCS that might allow a subsequent removal of a long-term LVAD or BiVAD is at present not yet possible. Although myocardial recovery rates tend to be higher in young patients with shorter history of HF before VAD therapy, less ventricular dilation and reduced myocardial fibrosis, neither the pre-implant ventricular size, geometry and EF, nor patient age, duration of HF, or the reduced fibrosis can reliably predict the extend and sustainability of possible cardiac recovery during LVAD support despite the fact that most centers try harder in these groups $(1,41,43)$. However, there is some indication that certain pre-implant genomic signals may help in better predicting HF reversibility on LVAD support $(41,86)$. The degree of HF-associated myocardial t-system remodeling and cardiac rotational mechanics also appeared promising for prediction of recovery before VAD implantation $(87,88)$. Further studies in this direction are reasonable, because prediction of possible VAD-promoted clinically relevant cardiac improvement already before the device implantation would facilitate the possible future use of VAD support also as a therapeutic strategy aimed to reverse HF.

\section{Conclusions and future objectives}

Although complete cardiac recovery after VAD implantation is rather seldom, systematic search for recovery is absolutely worthwhile because weaning from VADs is a validated and feasible clinical option which often provides good longterm results even if chronic HF has given rise to the need for emergent VAD support and even if recovery remains incomplete.

ECHO and RHC are the main cornerstones for assessment of myocardial recovery in VAD recipients. Based on the current experiences, prediction of postweaning freedom from HF recurrence in patients with varying degrees of unloading promoted cardiac recovery is challenging but certainly possible in most cases. Many parameters were found useful for assessment of recovery and for prediction of long-term weaning success but there is still no "golden standard" for recovery assessment and its prognostic evaluation. Further studies would be necessary to attain that goal.

Prediction of long-term stable ventricular reverse remodeling and functional improvement before VAD implantation in patients with a chronic cardiomyopathy is still not possible today and this is the major limitation for a potential future use of VADs as a targeted therapeutic strategy for reversal of HF before its end-stage has been reached. Considering the potential risks for complications related to the implantation and explantation of VADs as well as their prolonged use, such therapeutic strategy will necessitate a relatively accurate prediction of its efficacy.

There are several future goals and tasks that are needed for further improvement of the efficiency and safety of VADs as a bridge to recovery:

(I) More studies on myocardial recovery focused on changes at cellular and molecular levels to provide a platform for additional adjunctive therapies (pharmacologic and/or cell-based therapy, gene transfer etc.), focused on optimizing myocardial recovery and increasing the number of weaning candidates;

(II) Research on cellular and molecular level to gain more basic knowledge which might permit the detection of patients with the potential for cardiac recovery under mechanical support, already before VAD implantation;

(III) Search for possibilities to promote myocardial recovery allowing VADs explantation also in patients with chronic ischemic cardiomyopathy by promoting and enhancing angiogenesis and myocyte regeneration;

(IV) Further improvement of VAD technology at reducing the risk for complications related to VAD implantation and/or the prolonged use of mechanical circulatory support.

\section{Acknowledgments}

Funding: None.

\section{Footnote}

Provenance and Peer Review: This article was commissioned by the editorial office, Cardiovascular Diagnosis and Therapy 
for the series "Heart Failure in the Young and Old: Insights into Various Therapies". The article has undergone external peer review.

Conflicts of Interest: The authors have completed the ICMJE uniform disclosure form (available at http://dx.doi. org/10.21037/cdt-20-288). The series "Heart Failure in the Young and Old: Insights into Various Therapies" was commissioned by the editorial office without any funding or sponsorship. RH served as the unpaid Guest Editor of the series, and serves as an unpaid editorial board member of Cardiovascular Diagnosis and Therapy from Jul 2019 to Jun 2021. The authors have no other conflicts of interest to declare.

Ethical Statement: The authors are accountable for all aspects of the work in ensuring that questions related to the accuracy or integrity of any part of the work are appropriately investigated and resolved.

Open Access Statement: This is an Open Access article distributed in accordance with the Creative Commons Attribution-NonCommercial-NoDerivs 4.0 International License (CC BY-NC-ND 4.0), which permits the noncommercial replication and distribution of the article with the strict proviso that no changes or edits are made and the original work is properly cited (including links to both the formal publication through the relevant DOI and the license). See: https://creativecommons.org/licenses/by-nc-nd/4.0/.

\section{References}

1. Dandel M, Weng Y, Siniawski H, et al. Prediction of cardiac stability after weaning from left ventricular assist devices in patients with idiopathic dilated cardiomyopathy. Circulation 2008;118:S94-105.

2. Dandel M, Weng Y, Siniawski H, et al. Pre-explant stability of unloading-promoted cardiac improvement predicts outcome after weaning from ventricular assist devices. Circulation 2012;126:S9-19.

3. Dandel M, Hetzer R. Recovery of failing hearts by mechanical unloading: pathophysiologic insights and clinical relevance. American Heart Journal 2018;206:30-50.

4. Hall JL, Fermin DR, Birks EJ, et al. Clinical, molecular, and genomic changes in response to a left ventricular assist device. J Am Coll Cardiol 2011;57:641-52.

5. Mann DL, Barger MP, Burkhoff D. Myocardial recovery and the failing Heart. J Am Coll Cardiol 2012;60:2465-72.

6. Ambardekar AV, Walker JS, Walker LA, et al.

Incomplete recovery of myocyte contractile function despite improvement of myocardial architecture with left ventricular assist device support. Circ Heart Fail 2011;4:425-32.

7. Dandel M, Weng Y, Siniawski H, et al. Long-term results in patients with idiopathic dilated cardiomyopathy after weaning from assist devices. Circulation 2005;112:137-45.

8. Saito S, Matsumiya G, Sakaguchi T, et al. Cardiac fibrosis and cellular hypertrophy decrease the degree of reverse remodeling and improvement in cardiac function during left ventricular assist. J Heart Lung Transplant 2010;29:672-9.

9. Nakatani T, Sasako Y, Kumon K, et al. Long-term circulatory support to promote recovery from profound heart failure. ASAIO J 1995;41:M526-30.

10. Nakatani T, Takano H, Beppu S, et al. Practical assessment of natural heart function using echocardiography in mechanically assisted patients ASAIO Trans 1991;37:M420-1.

11. McBride LR, Naunheim KS, Fiore AC, et al. Clinical experience with 111 Thoratec ventricular assist devices. Ann Thorac Surg 1999;67:1233-8.

12. Farrar DJ, Holman WR, McBride LR, et al. Long-term follow up of Thoratec ventricular assist device bridge-torecovery patients successfully removed from support after recovery of ventricular function. J Heart Lung Transplant 2002;21:516-21.

13. Frazier OH, Radovancevic B, Abou-Avdi NL, et al. Ventricular remodeling after prolonged ventricular unloading "heart rest" experience with HeartMate left ventricular assist device (abstract) J Heart Lung transplant 1994;13:77.

14. Frazier OH, Benedict CR, Radovancevic B, et al. Improved left ventricular function after chronic left ventricular unloading. Ann Thorac Surg 1996;62:675-81; discussion 681-2.

15. Müller J, Wallukat G, Weng YG, et al. Weaning from mechanical cardiac support in patients with idiopathic dilated cardiomyopathy. Circulation 1997;96:542-9.

16. Frazier OH, Myers TJ. Left ventricular assist system as a bridge to myocardial recovery. Ann Thorac Surg 1999;68:734-41.

17. Mancini DM, Beniaminovitz A, Levin H, et al. Low incidence of myocardial recovery after left ventricular assist device implantation in patients with chronic heart 
failure. Circulation 1998;98:2383-9.

18. Birks EJ, Tansley PD, Hardy J, et al. Left ventricular assist device and drug therapy for the reversal of heart failure. $\mathrm{N}$ Engl J Med 2006;35 5:1873-84.

19. Dandel M, Knosalla C, Hetzer R. Contribution of ventricular assist devices to the recovery of failing hearts: a review and Berlin Heart Center experience. Eur J Heart Fail 2014;16:248-63.

20. Loebe M, Hennig E, Müller J, et al. Long-term mechanical circulatory support as a bridge to transplantation, for recovery from cardiomyopathy, and for permanent replacement. Eur J Cardiothorac Surg 1997;1 1 Suppl:S18-24.

21. Müller J, Wallukat G, Weng YG, et al. Weaning from mechanical support after complete recovery in patients with idiopathic dilated cardiomyopathy. Mechanical Circulatory Support@ C. Darmstadt: Steinkopff Verlag, 1997:93-108.

22. Hetzer R, Müller J, Weng Y, et al. Cardiac recovery in dilated cardiomyopathy by unloading with a left ventricular assist device. Ann Thorac Surg 1999;68:742-9.

23. Hetzer R, Müller JH, Weng Y, et al. Bridging-to-recovery. Ann Thorac Surg 2001;71:S109-13; discussion S114-5.

24. Birks EJ, George RS, Firouzi A, et al. Long-term outcomes of patients bridged to recovery versus patients bridged to transplantation. J Thorac Cardiovasc Surg 2012;144:190-6.

25. Birks EJ. Molecular changes after left ventricular assist device support for heart failure. Circulation Res 2013;113:777-91.

26. Drakos SG, Pagani FD, Lundberg MS, et al. Advancing the science of myocardial recovery with mechanical circulatory support. A Working Group of the National, Heart, Lung, and Blood Institute. JACC Basic Transl Sci 2017;2:335-40.

27. Segura AM, Frazier OH, Demirozu Z, et al. Histopathologic correlates of myocardial improvement in patients supported by a left ventricular assist device. Cardiovasc Pathol 2011;20:139-45.

28. Wever-Pinzon O, Drakos SG, McKellar SH, et al. Cardiac Recovery During Long-Term Left Ventricular Assist Device Support. J Am Coll Cardiol 2016;68:1540-53.

29. Dandel M, Hetzer R. Myocardial recovery during mechanical circulatory support: long-term outcome and elective ventricular assist device implantation to promote recovery as a treatment goal. Heart Lung Vessel 2015;7:289-96.
30. Jakovljevic DG, Yacoub MH, Schueler S, et al. Left ventricular assist device as a bridge to recovery for patients with advanced heart failure. J Am Coll Cardiol 2017;69:1924-33.

31. Stainback RF, Croitoru M, Hernandez A, et al. Echocardiographic evaluation of the JaRVik 2000 axialflow LVAD. Tex Heart Inst J 2005;32:263-70.

32. Dandel M, Hetzer R. Echocardiographic strain and strain rate imaging - Clinical applications. Int J Cardiol 2009;132:11-24.

33. Noor MR, Bowles C, Banner NR. Relationship between pump speed and exercise capacity during HeartMate II left ventricular assist device support: influence of residual left ventricular function. Eur J Heart Fail 2012;14:613-620.

34. Dandel M, Hetzer R. Temporary assist device support for the right ventricle: pre-implant and post-implant challenges. Heart Fail Rev 2018;23:157-71.

35. Lazar JF, Swartz MF, Schiralli MP, et al. Survival after left ventricular assist device with and without temporary right ventricular support. Ann Thorac Surg 2013;96:2155-9.

36. Dandel M, Hetzer R. Myocardial Recovery During Mechanical Circulatory Support: Weaning and Explantation Criteria. Heart Lung Vessel 2015;7:280-8.

37. Maybaum S, Mancini D, Xydas S, et al. Cardiac improvement during mechanical circulatory support: a prospective multicenter study of the LVAD Working Group. Circulation 2007;115:2497-505.

38. Selzman CH, Madden JL, Healy AH, et al. Bridge to removal: a paradigm shift for left ventricular assist device therapy. Ann Thorac Surg 2015;99:360-7.

39. Drakos SG, Kfoury AG, Stehlik J, et al. Bridge to recovery: understanding the disconnect between clinical and biological outcomes. Circulation 2012;126:230-41.

40. Miera O, Germann M. Choi MY, et al. Bridge to Recovery in Children on Ventricular Assist Devices-Protocol, Predictors of Recovery, and Long-Term Follow-Up. J Heart Lung Transplant 2018;37:1459-66.

41. Birks EJ, Selzman CH. Facilitating Myocardial recovery. In: Kirklin JK and Rogers JG (Second edition) Mechanical Circulatory Support. A Companion to Braunwald's Heart Disease. Philadelphia: Elsevier, 2020:223-41.

42. Birks EJ, George RS, Hedger M, et al. A Reversal of severe heart failure with a continuous-flow left ventricular assist device and pharmacological therapy: a prospective study. Circulation 2011;123:381-90.

43. Dandel M, Weng Y, Siniawski H, et al. Heart failure reversal by ventricular unloading in patients with chronic 
cardiomyopathy: criteria for weaning from ventricular assist devices. Eur Heart J 2011;32:1148-60.

44. Krabatsch T, Schweiger M, Dandel M, et al. Is bridge to recovery more likely with pulsatile left ventricular assist devices than with nonpulsatile-flow systems? Ann Thorac Surg 2011;91:1335-40.

45. Dandel M, Weng Y, Siniawski H, et al. Cardiac stability for more than 5 years after ventricular assist device removal: main predictors for long-term weaning success. J Heart Lung Transplant 2009;28:S208.

46. Hetzer R, Müller JH, Weng YG, et al. Midterm follow-up of patients who underwent removal of a left ventricular assist device after cardiac recovery from endstage dilated cardiomyopathy. J Thorac Cardiovasc Surg 2000;120:843-53.

47. Hetzer R, Dandel M, Knosalla C. Clinical, molecular, and genomic changes after left ventricular assist device implantation. J Am Coll Cardiol 2011;57:2459-60.

48. Hetzer R, Dandel M, Knosalla C. Left ventricular assist devices and drug therapy in heart failure $\mathrm{N}$ Engl J Med 2007;356:869-70.

49. Simon MA, Primack BA, Teuteberg J, et al. Left ventricular remodeling and myocardial recovery on mechanical circulatory support. J Card Fail 2010;16:99-105.

50. Estep JD, Stainback RF, Little SH, et al. The role of echocardiography and other imaging modalities in patients with left ventricular assist devices. JACC Cardiovasc Imaging 2010;3:1049-64.

51. Simon MA, Kormos RL, Murali S, et al. Myocardial recovery using ventricular assist devices: prevalence, clinical characteristics, and outcomes. Circulation 2005;112:I32-6.

52. Formica P, Murthy S, Edwards P, et al. A structured 3-step approach to evaluate cardiac recovery with continuous flow circulatory support. J Heart Lung Transplant 2010;29:1440-2.

53. Dandel M, Hetzer R. Evaluation of cardiac recovery in ventricular assist device recipients: particularities, reliability and practical challenges. Can J Cardiol 2019;35:523-34.

54. George RS, Yacoub MH, Tasca G, et al. Heamodynamic and echocardiographic responses to acute interruption of left ventricular assist device support - relevance to assessment of myocardial recovery. J Heart Lung Transplant 2007;26:967-73.

55. Cohen DG, Thomas JG, Freed BH, et al. Echocardiography and continuous flow left ventricular assist devices. JACC Heart Failure 2015;3:554-64.
56. Drakos SG, Wever-Pinzon O, Selzman CH, et al. Magnitude and time course of changes induced by continuous-flow left ventricular assist device unloading in chronic heart failure: insights into cardiac recovery. J Am Coll Cardiol 2013;61:1985-94.

57. George RS, Sabharwal NT, Webb C, et al. Echocardiographic assessment of flow across continuousflow ventricular assist devices at low speeds. J Heart Lung Transplant 2010;29:1245-52.

58. Dandel M, Hetzer R. Role of echocardiography in selection, implantation and management of left ventricular assist device therapy. Encyclopedia of Cardiovascular Research and Medicine. Elsevier, Amsterdam, Oxford, and Cambridge: 2018:327-44.

59. Vierecke J, Hernándes-Enriquez M, Dandel M, et al. Percutaneous balloon occlusion of the left ventricular assist device outflow cannula during right heart catheterization with pump-stop as part of the evaluation of myocardial recovery. J Heart Lung Transplant 2014;33:S156.

60. Khan T, Delgado RM, Radovancevic B, et al. Dobutamine stress echocardiography predicts myocardial improvement in patients supported by left ventricular assist devices (LVADs): hemodynamic and histologic evidence of improvement before LVAD explantation. J Heart Lung Transplant 2003;22:137-46.

61. Khan T, Okerberg K, Hernandez A, et al. Assessment of myocardial recovery using dobutamin stress echocardiography in LVAD patients. J Heart Lung Transplant 2001;20:202-3.

62. Andersen M, Gustafsson F, Madsen PL, et al. Hemodynamic stress echocardiography in patients supported with a continuous-flow left ventricular assist device. JACC Cardiovasc Imaging 2010;3:854-9.

63. Topkara VK, Chambers KT, Yang KC, et al. Functional significance of the discordance between transcriptional profile and left ventricular structure/function during reverse remodeling. JCI Insight 2016;1:e86038.

64. Metra M, Faggiano P, D'Aloia A, et al. Use of cardiopulmonary exercise testing with hemodynamic monitoring in the prognostic assessment of ambulatory patients with chronic heart failure. J Am Coll Cardiol 1999;33:943-50.

65. Wilson JR, Rayos G, Yeoh TK, et al. Dissociation between peak exercise oxygen consumption and hemodynamic dysfunction in potential heart transplant candidates. J Am Coll Cardiol 1995;26:429-35.

66. Gupta DK, Skali H, Rivero J, et al. Assessment of 
myocardial viability and left ventricular function in patients supported by a left ventricular assist device. J Heart Lung Transplant 2014;33:372-81.

67. Stainback RF, Estep JD, Agler DA, et al. Echocardiography in the Management of Patients with Left Ventricular Assist Devices: Recommendations from the American Society of Echocardiography. J Am Soc Echocardiogr 2015;28:853-909.

68. Jasaityte R, Dandel M, Lehmkuhl H, et al. Prediction of short-term outcomes in patients with idiopathic dilated cardiomyopathy referred for transplantation using standard echocardiography and strain imaging. Transplant Proc 2009;41:277-80.

69. Dandel M, Potapov E, Vierecke J, et al. Low diastolic arterial pressure during pre-explant off-pump trials can induce misleading overestimation of left ventricular function with potential risk for early recurrence of heart failure after left ventricular assist device removal. Circulation 2012;126:A9754.

70. Hetzer R, Dandel M. Early detection of left ventricular dysfunction in patients with mitral regurgitation due to flail leaflet is still a challenge. Eur Heart J 2011:32:665-67.

71. Liden H, Karason K, Bergh CH, et al. The feasibility of left ventricular mechanical support as a bridge to cardiac recovery. Eur J Heart Fail 2007;9:525-30.

72. Cleveland JC, Naftel DC, Reece TB, et al. SuRVival after biventricular assist device implantation: an analysis of the interagency registry for mechanical assisted circulatory support database. J Heart Lung Transplant 2011;30:862-69.

73. Shehab S, Macdonald PS, Keogh AM, et al. Long-term biventricular HeartWare ventricular assist device support-Case series of right atrial and right ventricular implantation outcomes. J Heart Lung Transplant 2016;35:466-73.

74. Takeda K, Naka Y, Yang JA, et al. Timing of temporary right ventricular assist device insertion for severe right heart failure after left ventricular assist device implantation. ASAIO J 2013;59:564-9.

75. Lazar JF, Swartz MF, Schiralli P, et al. Survival after left ventricular assist device with and without temporary right ventricular support. Ann Thorac Surg 2013;96:2155-9.

76. Deschka H, Holthaus AJ, Sindermann JR. Can perioperative right ventricular support prevent postoperative right heart failure in patients with biventricular dysfunction undergoing left ventricular assist device implantation? J Cardiothorac Vasc Anesth 2016;30:619-26.

77. Leidenfrost J, Prasad S, Itoh A, et al. Right ventricular assist device with membrane oxygenator support for right ventricular failure following implantable left ventricular assist device placement. Eur J Cardiothorac Surg 2016;49:73-7.

78. Houston BA, Kalathiya RJ, Hsu S, et al. Right ventricular afterload sensitivity dramatically increases after left ventricular device implantation: A multicenter hemodynamic analysis J Heart Lung Transplant 2016;35:868-76.

79. Saeed D, Maxhera B, Kamiya H, et al. Alternative right ventricular assist device implantation technique for patients with preoperative right ventricular failure. J Thorac Cardiovasc Surg 2015;149:927-32.

80. Dandel M, Potapov E, Krabatsch T, et al. Right ventricular assist device removal in patients with apparently unloading-promoted improvement of right ventricular function: Criteria for weaning decisions. Circulation 2011;124:17718.

81. Akhter SA, Jeevanandam V. Special clinical settings for mechanical circulatory support. In: Kormos RL, Miller LW (eds.) Mechanical circulatory support. Philadelphia: Elsevier Saunders, 2012:118-27.

82. Saffarzadeh A, Bonde P. Options for temporary mechanical circulatory support. J Thorac Dis 2015;7:2102-11.

83. Dandel M, Potapov E, Krabatsch T, et al. Load dependency of right ventricular performance is a major factor to be considered in decision making before ventricular assist device implantation. Circulation 2013;128:S14-23.

84. Dandel M, Knosalla C, Kemper D, et al. Assessment of right ventricular adaptability to loading conditions can improve the timing of listing to transplantation in patients with pulmonary arterial hypertension. J Heart Lung Transplant 2015;34:319-28.

85. Dandel M, Weng Y, Siniawski H, et al. Time course of unloading induced cardiac improvement in patients with chronic non-ischemic cardiomyopathy predicts the outcome after weaning from ventricular assist devices. J Heart Lung Transplant 2010;29:S64-65.

86. Yang KC, Yamada KA, Patel AY, et al. Deep RNA sequencing reveals dynamic regulation of myocardial noncoding RNAs in failing human heart and remodeling with mechanical circulatory support. Circulation 2014;129:1009-21.

87. Seidel T, Navankasattusas S, Ahmad A, et al. Sheet-like remodeling of the transverse tubular system in human heart failure impairs excitation-contraction coupling and 
functional recovery by mechanical unloading. Circulation 2017;135:1632-45.

88. Bonios MJ, Koliopoulou A, Wever-Pinzon O, et al. Cardiac Rotational Mechanics As a Predictor of Myocardial

Cite this article as: Dandel M, Javier MFDM, Javier Delmo EM, Loebe M, Hetzer R. Weaning from ventricular assist device support after recovery from left ventricular failure with or without secondary right ventricular failure. Cardiovasc Diagn Ther 2021;11(1):226-242. doi: 10.21037/cdt-20-288
Recovery in Heart Failure Patients Undergoing Chronic Mechanical Circulatory Support: A Pilot Study. Circ Cardiovasc Imaging 2018;11:e007117. 